Recepción: 20 / 01 / 2017

Aceptación: 20 / 02 / 2017

\title{
Terapia antifímica empleada en pacientes con coinfección Tuberculosis/VIH
}

\author{
Anti-chemical therapy used in patients with tuberculosis / HIV coinfection
}

\section{Antifimica terapia utilizada em pacientes com tuberculose / VIH}

Roció M. Verduga Monar ${ }^{\text {II }}$ rocioverduga1925@hotmail.com

Frank E. Palma Mendieta III frank_palma_mendieta9@hotmail.com

María G. Ruiz Ortega IV gisella.ruizo@ug.edu.ec

Correspondencia: md.eddyabril2621@hotmail.com

\footnotetext{
Diploma Superior en Atención Primaria en Salud; Medico, Universidad de Guayaquil, Guayaquil, Ecuador.

II. Magister en Docencia Universitaria e Investigación Educativa; Diplomado Epidemiologia en Acción; Diplomado en Docencia Superior; Licenciada en Enfermería, Universidad de Guayaquil, Guayaquil, Ecuador.

III. Médico, Universidad de Guayaquil, Guayaquil, Ecuador.

Iv. Magister en Gerencia en Salud para el Desarrollo Local; Magister en atención Primaria y Clínica Infantil; Especialista en Cirugía Plástica Reconstructiva y Estética; Diploma Superior de Cuarto Nivel en Desarrollo Local y Salud; Doctor en Medicina y Cirugía; Universidad de Guayaquil, Guayaquil, Ecuador.
} 
Eddy J. Abril-Plata; Roció M. Verduga-Monar; Frank E. Palma-Mendieta; María G. Ruiz-Ortega

\section{Resumen}

Objetivo: Describir la terapia antifímica empleada en pacientes con coinfección TB/VIH en el territorio de la Zona 8 Salud de Ecuador, (cantones de Guayaquil, Durán y Samborondón), período 2015. Material y métodos: estudio observacional, analítico, retrospectivo y descriptivo. Se recogieron 277 casos con diagnóstico de coinfección por TB/VIH como muestra de estudio, revisando los Sistemas de Información de la Estrategia de Prevención y Control de la Coordinación Zona 8 Salud que corresponde a los cantones Guayaquil, Duran y Samborondón año 2015. Resultados: De un total de 2.390 casos de tuberculosis (todas las formas, excluyendo los casos de Mycobacterium tuberculosis resistentes), se incluyeron en el estudio 277 pacientes coinfectados con TB/VIH (11.6\%), de los cuales el 99\% realizaban tratamiento antifímico basados en una combinación de Rifampicina, Isoniacida, Pirazinamida y Etambutol, durante 9 a 12 meses, según el caso; de forma diaria, bajo DOTS y seguido de Tratamiento Antirretroviral (TAR). La mayoría de ellos hombres $(77.9 \%)$ con una mediana de edad de 29,5 años. El tratamiento concomitante ha influido en la reducción del índice de mortalidad y deserciones de tratamientos. Conclusiones: Se pudo determinar que la terapia antifímica conjuntamente con el TAR indistintamente de los CD4 era la prioridad en el manejo de pacientes coinfectados TB/VIH en los establecimientos de salud de la población en estudio.

Palabras clave: Terapia antifímica; coinfección TB/VIH; tratamiento antirretroviral; mortalidad. 


\section{Abstract}

Objective: To describe the antifimic. therapy used in patients with TB / HIV in the territory of the Zone 8 Health (Guayaquil, Durán and Samborondón) in the period 2015. Material and Methods: observational, analytical and descriptive study. Being a finite population, fully corresponding determined 277 cases TB / HIV as study sample, reviewing the Information System Strategy for Prevention and Control Area Coordination 8 Health corresponding to Guayaquil, Duran and Samborondón cantons - Ecuador 2015. Results: Out of a total of 2,390 cases of tuberculosis (all forms, excluding cases with resistant Mycobacterium tuberculosis), were included in the study 277 patients co-infected with TB / HIV (11.6\%), of which $99 \%$ were under treatment for tuberculosis based a combination of rifampin, isoniazid, pyrazinamide and ethambutol for 9 to 12 months, as appropriate; daily, followed by DOTS and antiretroviral therapy (ART). Most of them men (77.9\%) with a median age of 29.5 years. Concomitant treatment has influenced the reduction of mortality rate and defections treatments. Conclusions: It was determined that the antifimic therapy in conjunction with ART regardless of CD4 was the priority in the management of patients co-infected TB / HIV in health facilities of the study population.

Key words: Antifimic therapy; co-infection; antiretroviral therapy; mortality. 
Eddy J. Abril-Plata; Roció M. Verduga-Monar; Frank E. Palma-Mendieta; María G. Ruiz-Ortega

\section{Resumo}

Objetivo: descrever o antifimic. Terapia utilizada em pacientes com TB / HIV no território da Saúde da Zona 8 (Guayaquil, Durán e Samborondón) no período 2015. Material e Métodos: estudo observacional, analítico e descritivo. Sendo uma população finita, totalmente correspondente, determinou 277 casos de TB / HIV como amostra do estudo, revisando a Estratégia do Sistema de Informação para a Coordenação da Área de Controle e Prevenção 8 Saúde correspondente aos cantões de Guayaquil, Duran e Samborondón - Equador 2015. Resultados: De um total de 2.390 Os casos de tuberculose (todas as formas, excluindo casos com Mycobacterium tuberculosis resistente), foram incluídos no estudo 277 pacientes co-infectados com TB / HIV (11,6\%), dos quais 99\% estavam sob tratamento para tuberculose com base em uma combinação de rifampicina, isoniazida , Pirazinamida e etambutol durante 9 a 12 meses, conforme apropriado; Diariamente, seguido de DOTS e terapia anti-retroviral (ART). A maioria deles $(77,9 \%)$ com idade média de 29,5 anos. O tratamento concomitante tem influenciado a redução da taxa de mortalidade e dos tratamentos de defecções. Conclusões: Foi determinado que a terapia antifimica em conjunto com ART, independentemente do $\mathrm{CD} 4$, foi a prioridade no tratamento de pacientes co-infectados TB / HIV em instalações de saúde da população estudada.

Palavras chave: Terapia antifimica; Co-infecção; Terapia anti-retroviral; mortalidade. 


\section{Introducción.}

La aparición del Síndrome de inmunodeficiencia adquirida (SIDA) ha cambiado radicalmente la epidemiología de la tuberculosis y está haciendo aún más difícil su control. La tuberculosis se ha constituido en una de las más viejas endemias que afectan a la humanidad, y la más reciente pandemia producida por el virus de la inmunodeficiencia humana (VIH), potenciando sus efectos hasta convertirse en la primera causa de muerte en el mundo.

Es de conocimiento general que el VIH determina una disminución de la inmunidad celular medida por los linfocitos CD4 de los cuales depende la inmunidad protectora frente a la tuberculosis. Así no es raro que ambas enfermedades se potencien. La degeneración de los linfocitos CD4 y la inmunosupresión generalizada, son los principales factores que hacen que la inmunopatología del SIDA sea un proceso complejo. A pesar de los esfuerzos que se han hecho en cuanto al manejo de estos pacientes las infecciones oportunistas y en especial la tuberculosis sigue siendo la principal causa de morbimortalidad en el mundo.

El primer paso que ha de darse para que el VIH pueda infectar una célula humana es que este en su superficie el receptor CD4 que es reconocido por el GP.120 de la envoltura viral.

La asociación tuberculosis-SIDA empeora el pronóstico de ambas enfermedades. Sin embargo, la introducción de los llamados TARV ha cambiado este desalentador panorama. Hasta hace más de diez años casi el 50\% de los pacientes que presentaban la comorbilidad morían antes de terminar el tratamiento de tuberculosis, siendo la causa principal el SIDA y otras enfermedades oportunistas como la TB que lo acompañaban, ya que la tuberculosis determina un aumento del TNF- $\alpha$ que puede acelerar la replicación del virus. Estudios recientes demuestran que la mortalidad de los pacientes infectados con VIH puede aumentar hasta cinco veces cuando el diagnostico se 
Eddy J. Abril-Plata; Roció M. Verduga-Monar; Frank E. Palma-Mendieta; María G. Ruiz-Ortega

realiza tardíamente. Por consiguiente, la identificación temprana de la infección VIH y la adecuada instauración del TARV tienen una influencia decisiva tanto en el pronóstico del SIDA como en la posibilidad que tienen estos enfermos de desarrollar tuberculosis.

Con los tratamientos actuales de ambas enfermedades, la letalidad de la asociación tuberculosis-Sida puede reducirse hasta el 1 al 5\% es decir cifras no muy diferentes de las que tienen los pacientes infectados exclusivamente Mycobacterium tuberculosis.

De acuerdo a las normas del País - Ecuador los pacientes coinfectados con TB/ VIH reciben las mismas pautas de tratamiento antifímico que los no coinfectados (2HRZE/HR), con ciertas modificaciones en el tiempo (9 meses y 12 meses según el caso) y la frecuencia (diaria) en la segunda fase, siendo este prioritario, seguido del TAR e indistintamente de sus CD4. Cabe destacar que el éxito del tratamiento en estos como en los otros enfermos en nuestro medio depende el $100 \%$ del DOTS tanto en la fase de inducción como en la de mantenimiento.

El presente trabajo tiene como objetivo describir la terapia antifímica empleada en pacientes con coinfección TB/VIH en el territorio de la Zona 8 Salud en el período 2015.

\section{Materiales y métodos.}

El método a utilizar es el estadístico, descriptivo, analítico retrospectivo y para el análisis de datos el sistema estadístico de EXCEL.

\section{Población}

Los pacientes diagnosticados con coinfección VIH/TB en la Coordinación Zona 8 Salud.

\section{MUESTRA}


Por ser un estudio Epidemiológico con una población finita delimitada se toma como muestra la totalidad de la población afectada por TB/VIH en la Zona 8 Salud que corresponde a Guayaquil, Duran y Samborondón.

\section{Pacientes y métodos}

\section{Pacientes y diseño del estudio}

Ámbito del estudio. El estudio incluyó a pacientes ambulatorios y hospitalizados con coinfección tuberculosis VIH que recibieron tratamiento en los establecimientos de salud de la Coordinación Zonal 8 Salud-Ecuador, que incluye los cantones de Guayaquil, Duran y Samborondón, durante el periodo de tiempo comprendido entre el 02 de enero a diciembre de 2015.

La Zona 8 en estudio cuenta con una población de 2.956.768 habitantes. Para el 2015 registra 2.390 casos de tuberculosis en todas las formas, es decir una tasa de prevalencia de 80.83 casos por 100000 habitantes, de estos 2252 son casos nuevos de tuberculosis (tasa de incidencia de 76 por 100000 habitantes), 1723 son casos nuevos BK+ (tasa de 58.3 por 100.000 hab),

Diseño del estudio: el estudio es observacional descriptivo y retrospectivo (se analizó un periodo de un año)

\section{Resultados.}

Se revisaron y analizaron las estadísticas del Sistema de Información (SI) de la Estrategia de Tuberculosis Zona 8 Salud 2015, encontrando 2.390 casos de tuberculosis en todas sus formas, es decir una tasa de prevalencia de 80.83 casos por 100000 habitantes, de estos 2252 son casos nuevos de tuberculosis (tasa de incidencia de 76 por 
Eddy J. Abril-Plata; Roció M. Verduga-Monar; Frank E. Palma-Mendieta; María G. Ruiz-Ortega

100000 habitantes), 1723 son casos nuevos $\mathrm{BK}+$ (tasa de 58.3 por 100.000 hab),

Del total (2390) casos de TB. De la Zona 8 Salud 277 (11.58\%) corresponden a TB/VIH., y de estos $77.9 \%$ son hombres.

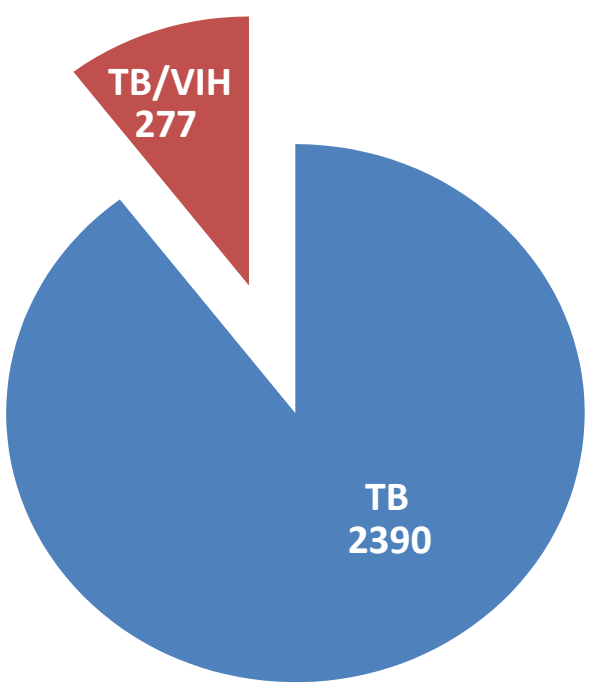

Gráfico $N^{\circ}$ 1.- Coinfeccion TB/VIH zona 8 salud: Guayaquil, Duran y Samborondon año 2015

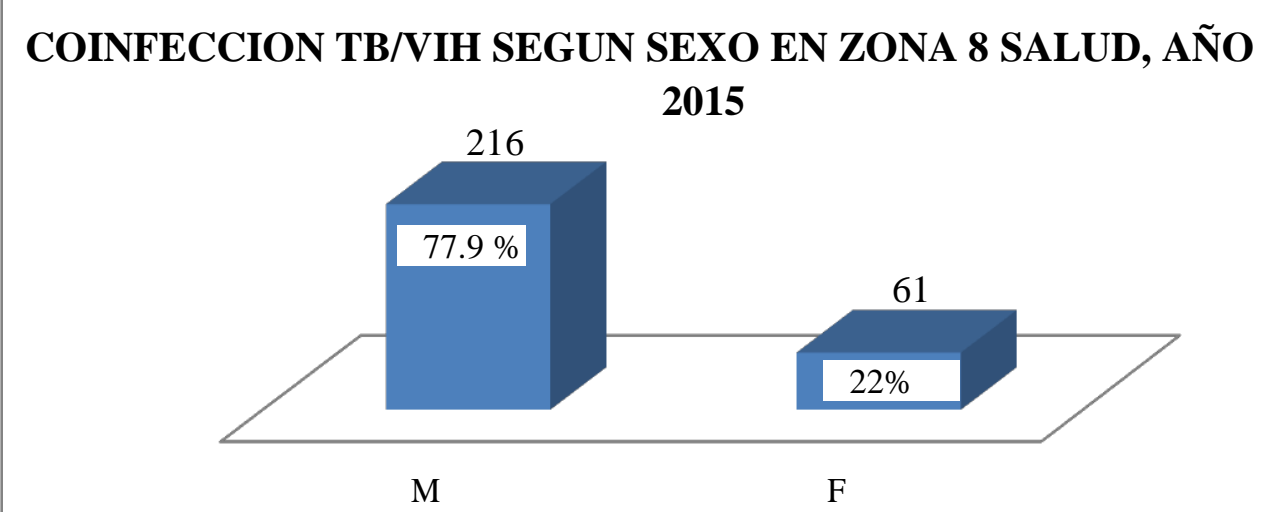

Fuente: Sistema de Información ECTB Zona 8

Gráfico $\mathrm{N}^{\circ}$ 2.- Coinfeccion TB/VIH 
El grupo etáreo más afectado es el comprendido entre 25 y 34 años del sexo masculino conforme muestra el grafico No3. Y la región geográfica con mayor afectación es el Distrito 09D04 y 09D07 correspondiendo a zonas urbano marginales de gran hacinamiento y pobreza ver Tabla 1.

\begin{tabular}{|c|c|c|c|c|c|c|c|c|c|c|c|c|c|c|}
\hline \multirow[t]{2}{*}{ Descripción } & \multirow{2}{*}{$\begin{array}{c}0-4 \\
M\end{array}$} & \multicolumn{2}{|c|}{$5-14$} & \multicolumn{2}{|c|}{$15-24$} & \multicolumn{2}{|c|}{$25-34$} & \multicolumn{2}{|c|}{$35-44$} & \multicolumn{2}{|c|}{$45-54$} & \multicolumn{2}{|c|}{$55-64$} & \multirow{2}{*}{$\begin{array}{c}64 \mathrm{Y} \\
\text { MAS } \\
\mathrm{F}\end{array}$} \\
\hline & & $\mathbf{F}$ & $\mathbf{M}$ & $\mathbf{F}$ & $\mathbf{M}$ & $\mathbf{F}$ & $\mathbf{M}$ & $\mathbf{F}$ & $\mathbf{M}$ & $\mathbf{F}$ & $\mathbf{M}$ & $\mathbf{F}$ & $\mathbf{M}$ & \\
\hline TOTAL NUEVOS TB/VIH & 0 & 0 & 5 & 2 & 21 & 15 & 47 & 28 & 27 & 6 & 7 & 1 & 3 & 0 \\
\hline TOTAL ANTES TRATADOS TB/VIH & 0 & 0 & 0 & 0 & 3 & 1 & 6 & 0 & 4 & 0 & 0 & 0 & 0 & 0 \\
\hline TOTAL TODAS FORMAS & 0 & 0 & 5 & 2 & 24 & 16 & 53 & 28 & 31 & 6 & 7 & 1 & 3 & 0 \\
\hline
\end{tabular}

Tabla $N^{\circ}$ 1.- Coinfectados TB/VIH por grupo etario y sexo. Zona 8 año 2015

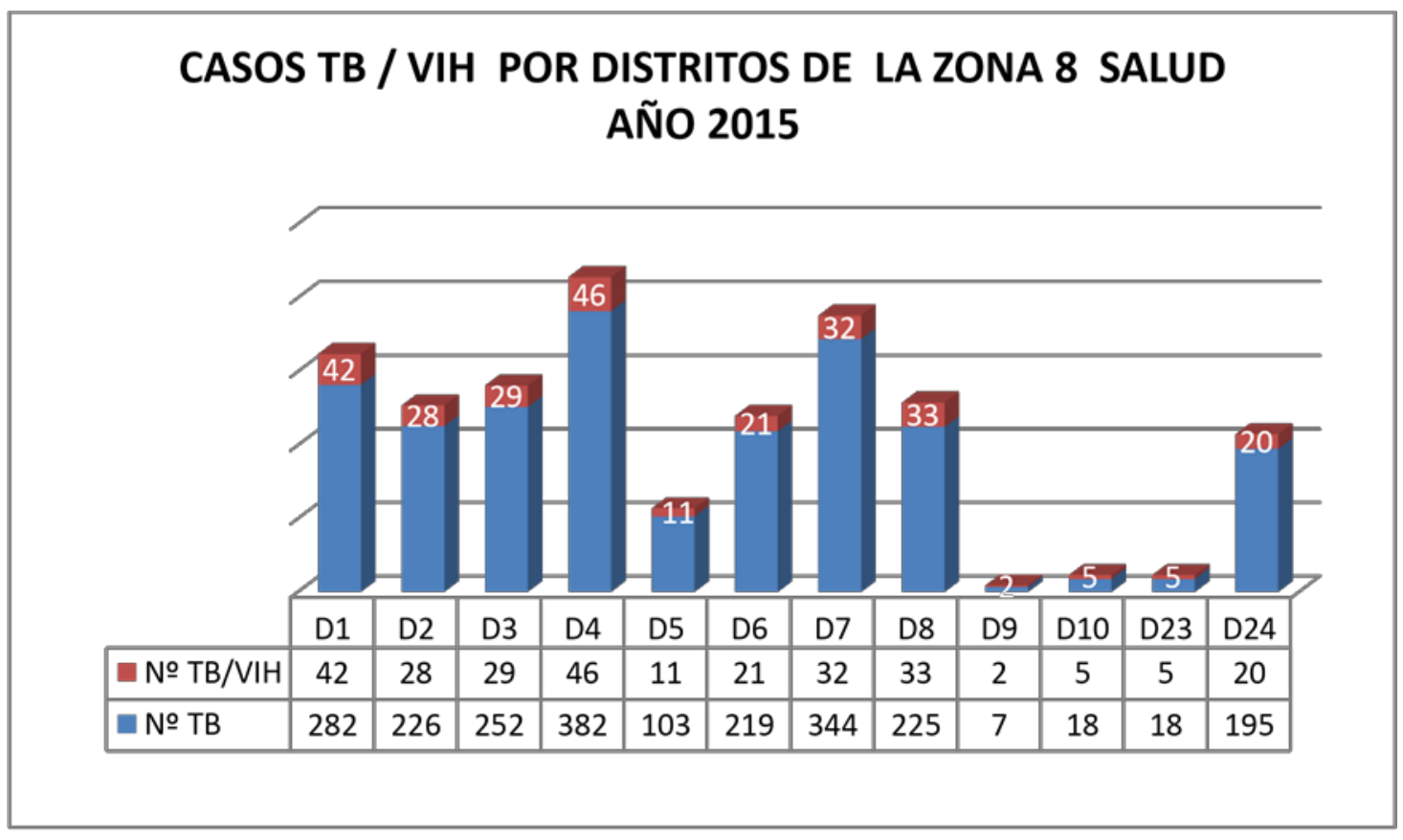

\section{Gráfico $N^{\circ}$ 3.- Casos TB/VIH}

El gráfico No 3 muestra la carga de tuberculosis por distritos de los cantones de Guayaquil, Samborondón y Duran de la Zona 8 salud y puede observarse que los casos están mayormente concentrados en la ciudad de Guayaquil (distritos D1 al D10), seguido del Cantón Duran (D 24) con 
Eddy J. Abril-Plata; Roció M. Verduga-Monar; Frank E. Palma-Mendieta; María G. Ruiz-Ortega

185 casos de Tuberculosis y 20 pacientes coinfectados, y en tercer lugar el Cantón Samborondón (D23) con 18 casos de tuberculosis y 5 pacientes coinfectados.

\section{COINFECCION TB/VIH CON TRATAMIENTOS PARA \\ TUBERCULOSIS EN LOS CANTONES DE LA ZONA 8 SALUD \\ AÑO 2015}

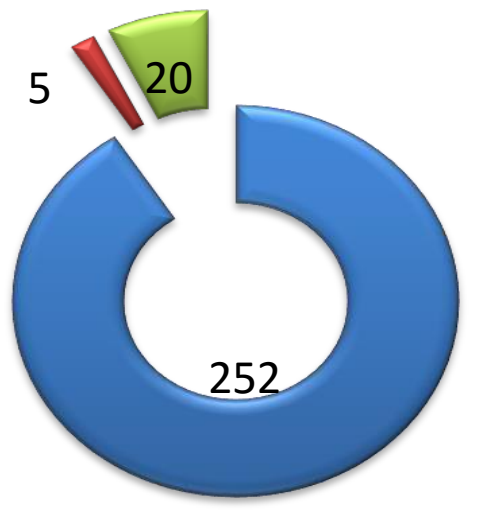

Guayaquil

Samborondon

$\square$ Duran

\section{Gráfico $N^{\circ}$ 4.- COINFECCION TB/VIH}

Del grupo en estudio (277 pacientes coinfectados) el 100\% recibieron tratamientos antituberculosis (grafico 4) y $275(99.3 \%)$ lo hicieron concomitantemente con TAR (Gráfico 5). A 2 de ellos se les suspendió TAR por reacciones adversas. Los pacientes que recibieron la terapia combinada han mostrado un éxito de tratamiento del 90\% (250). Gráfico No 6. 


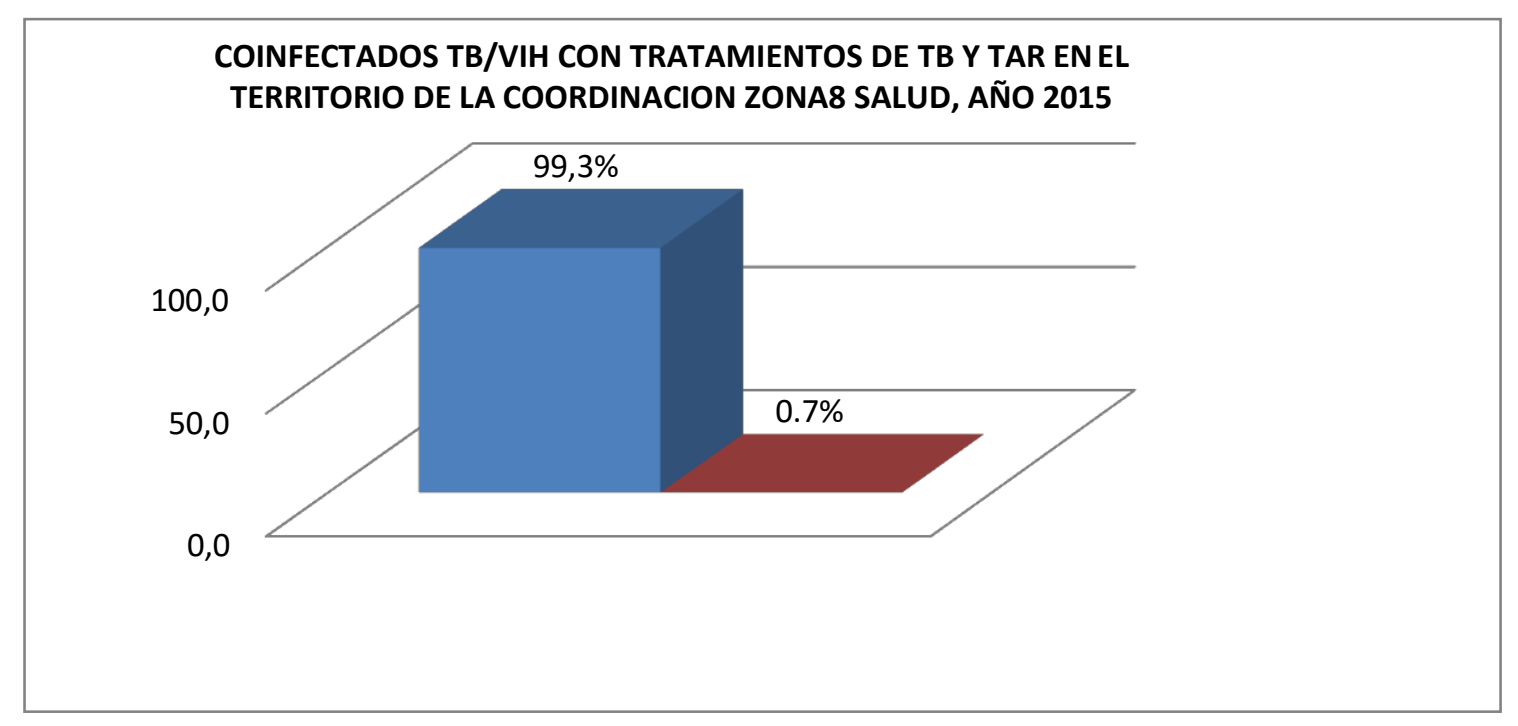

Gráfico $N^{\circ}$ 5.- COINFECCION TB/VIH

EXITO DE TRATAMIENTO DE LOS CASOS DE COINFECCION TB/VIH QUE RECIBIERON TERAPIA ANTIFIMICA Y TAR PERIODO 2015

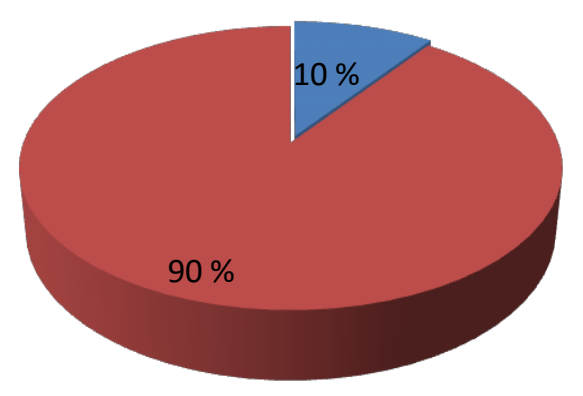

- FRACASO DE TRATAMIENTO

- ÉXITO DE TTO

Gráfico $N^{\circ}$ 6. - EXITO DE TRATAMIENTO DE LOS CASOS DE COINFECCION TB/VIH 
Eddy J. Abril-Plata; Roció M. Verduga-Monar; Frank E. Palma-Mendieta; María G. Ruiz-Ortega

\section{Discusión}

Los 277 pacientes coinfectados con tuberculosis y VIH objeto de este estudio presentaron características particulares que es importante señalar.

El grupo de pacientes que se seleccionó para el estudio estuvo compuesto mayoritariamente por varones (93) con una mediana de edad de 29.5 (percentil 25-percentil 75) y pertenecían mayoritariamente al Cantón Guayaquil (90.9\%) y dentro de este la mayor concentración de casos de TB y TB/VIH está en zonas urbano marginales caracterizada por pobreza y hacinamiento, como son en el orden de mayor a menor incidencia los distritos 4, 7 y 8. El elevado riesgo de transmisión de la infección por tuberculosis en estas zonas es evidente y se agrava frente a la incidencia del VIH. Es importante destacar que el país de Ecuador tienen una normativa bien diseñada para el manejo de la coinfección TB /VIH.

La terapia antifímica administrada a los pacientes con coinfección TB/VIH se basa en esquemas terapéuticos estandarizados y normados para el país, y consiste en la combinación de cuatro fármacos de primera línea (ISONIACIDA, RIFAMPICINA, PIRAZINAMIDA, ETAMBUTOL) asociados a la terapia antirretroviral TAR, independientemente de la carga viral. Las evidencias científicas demuestran que una tuberculosis activa conlleva un descenso en el recuento de CD4 y un aumento de la carga viral lo que favorece la progresión de la infección por VIH y empeora la evolución de la propia infección tuberculosa.

Es importante indicar que el TAR se utiliza para lograr la supresión máxima del ARN viral en el plasma, prevención de enfermedades oportunistas y la restitución inmunológica. 
Los esquemas de preferencia que incluyen en Ecuador son: 2 ITRN + 1 TRNN, el esquema de elección es TDF + FTC (o 3 TC) + EFV. Si llegase a existir alguna contraindicación para el uso de TDF, las alternativas serían:

$$
\begin{gathered}
\mathrm{ABC}+3 \mathrm{TC}+\mathrm{EFV} \\
\mathrm{AZT}+3 \mathrm{TC}+\mathrm{EFV}
\end{gathered}
$$

\section{Conclusiones.}

Los pacientes (275) que recibieron la terapia combinada han mostrado un éxito de tratamiento del $90 \%(250)$ y el $10 \%$ restante corresponde a los abandonos (3\%), fracasos (3\%) y fallecidos $(4 \%)$.

Es importante destacar que la asociación de los tratamientos (antifimicos y TAR) ha dado lugar en nuestro territorio a una reducción de los abandonos de tratamiento y de los fracasos.

\section{Recomendaciones.}

Seguir las directrices de la Organización Mundial de la Salud (OMS) y del país para pacientes con tuberculosis VIH-positivos que se basan en iniciar tratamiento anti-TB bajo DOTS primero, seguido de ARV tan pronto como sea posible, dentro de las 8 semanas del inicio del tratamiento anti-TB independientemente de los niveles de linfocitos CD4+.

Se recomienda la profilaxis con TMP/SMX en las personas con infección por VIH y CD4 < a 200 cel./mm3, lo largo de todo el tratamiento antituberculoso.

Investigar la serología VIH en todos los casos de tuberculosis y mantener una adecuada coordinación interprogramática entre las estrategias de tuberculosis y VIH, y viceversa con la 
Eddy J. Abril-Plata; Roció M. Verduga-Monar; Frank E. Palma-Mendieta; María G. Ruiz-Ortega

finalidad de identificar precozmente la presencia de la enfermedad tuberculosa en los infectados por

VIH, y la consideración de la terapia preventiva con isoniacida en los pacientes con infección latente por Micobacterium tuberculosis, previo descarte de enfermedad tuberculosa.

\section{Abreviaturas.}

VIH: Virus inmunodeficiencia humana

SIDA: Síndrome de inmunodeficiencia adquirida

TB: Tuberculosis

TARV: Tratamiento antirretroviral

OMS: Organización Mundial de la Salud

DOTS: Directly Observed Treatment Short/ Estrategia de tratamiento acortado directamente observado MSP: Ministerio de Salud Publican

WHO: World Health Organization

EPCTB: Estrategia de Prevención y Control de la Tuberculosis

EPC: Estrategia de Prevención y Control

ITRN: Inhibidor de la Transcriptasa inversa Análogo Nucleósido

TRNN: Inhibidor de la Transcriptasa inversa Análogo No Nucleósido 


\section{Bibliografía.}

Ministerio de Salud Pública. (2013). Modelo de Atención Integral del Sistema Nacional de Salud. Ecuador: MSP. pp. 23-97

Ministerio de Salud Pública. (2010). Manual de Normas y Procedimientos para el Control de la Tuberculosis. Ecuador: MSP. pp. 17-34

Caminero, J. (2003). Guía de la Tuberculosis para Médicos Especialistas. París: UICTER. pp. 1-39

Farga, V., Caminero J. (2011). Tuberculosis. Santiago: Mediterráneo. pp. 3-31

Williams, G., Alarcón E., \& Sebek M. (2007). Prácticas Óptimas en la Atención a los pacientes con Tuberculosis. París: UICTER. pp. 1-3

Enarson, D. (2000). Manejo de la Tuberculosis. París: WHO. pp. 2-6

INSPI. (2012). Manual de Normas Técnicas y Procedimientos para el Diagnóstico de la Tuberculosis por Microscopía Directa. Ecuador: MSP. pp. 1013

MSP/ACDI/ACP. (2011). Evaluación del Programa de Control de la Tuberculosis. Ecuador: MSP. pp. 12-15 OMS/OPS. (1999). Guía de Enfermería para la aplicación de la Estrategia DOTS/TAES. Perú: OMS. pp. 812

OPS/OMS/MSP. (2005). Informes de evaluación conjunta del PCT. Ecuador: MSP. pp. 15-17

Rieder, H. (2004). Bases Epidemiológicas para el Control de la Tuberculosis. París: UICTER. pp. 12-13

Dubos, Pines. (2010). Salud y Enfermedad. México: Time Life. pp. 8-9

Frieden. (2009). Tuberculosis. Washington: OPS. pp. 7-11

Kendall. (2009). Tuberculosis. Estados Unidos: UNICEF. p.5

Frías. (2011). Salud Pública y Educación para la Salud. Barcelona: Elzevir. p.6

Cabrera, Rodríguez \& Freimiento. (2008). Manual de Enfermedades Respiratorias. México: La Unión. p. 8

Sontang, S. (2009). La Enfermedad y sus metáforas. México: Editorial Primera. p. 4

Toman, K. (2008). Tuberculosis. México: OMS. pp. 10-11

Sontang, S. (2009). La Enfermedad y sus metáforas. México: Editorial Primera. p. 7

Arias, F. (2012). El proyecto de investigación. Caracas: Episteme. p 35

Sabino, C. (1986). El proceso de investigación. Caracas: Humanitas. p 77

Arias, F. (1999). Proyecto de la Investigación. Caracas: Episteme. p 98

Balestrini, M. (1997). Metodología de la investigación. Venezuela: Panapo. p 56

Castro, V. (2009). Recolección de la Información. Santiago: Mediterráneo. p 58 
Eddy J. Abril-Plata; Roció M. Verduga-Monar; Frank E. Palma-Mendieta; María G. Ruiz-Ortega

Hernández, R. (2003). Metodología de la investigación. México: Mc Graw Hill. p 44

Carretero, M. (2010). Paradigmas educativos. México. Editorial Limusa.

García Duque. Jairo. (2007) “Estrategias Metodológicas”, Editorial Morai. Caracas. p. 23

Navarro B, Néstor. "Seminario Investigativo" Universidad Javeriana. Ediciones Granica. Bogotá, Colombia. p. 36 